\title{
Cognitive appraisals and emotional status following a spinal cord injury in post-acute rehabilitation
}

\author{
Rebecca Eaton $^{1} \cdot$ Kevin Jones ${ }^{1} \cdot$ Jane Duff $^{1}$
}

Received: 25 August 2017 / Revised: 21 March 2018 / Accepted: 28 March 2018 / Published online: 12 June 2018

(c) International Spinal Cord Society 2018

\begin{abstract}
Study design Retrospective, cross-sectional study.

Objectives To investigate the factor structure of the ADAPSS-short form (ADAPSS-SF) in an acutely injured SCI population and to assess the relationship between cognitive appraisals made in the initial phase of rehabilitation and the experience of anxiety and depression.

Setting National Spinal Injuries Centre, UK.

Method Participants were acutely injured patients admitted to the NSIC over 35 months. Cognitive appraisals were measured using the ADAPSS-SF; psychological distress was measured using the HADS. Individual profiles, including demographics and injury characteristics, were collected.

Results Principle Component Analysis with oblique rotation demonstrated a coherent two-factor structure of the ADAPSSSF: resilience and loss. Correlational analysis found that individuals who negatively appraised their injury were more likely to report lower mood. Findings identified four vulnerable subgroups that were more likely to negatively appraise their injury: females, individuals older at the time of SCI, individuals with AIS-A injuries and individuals whose SCI was acquired through assault. Hierarchical regression analysis reported that resilience and loss factors were significant predictors of depression. Gender, resilience and loss factors were significant predictors of anxiety. Cognitive appraisals accounted for more variance in mood above biological markers.

Conclusions Findings support a two-factor structure and validity of ADAPSS-SF in acute SCI rehabilitation. The study provides support for the role of cognitive appraisals in psychological adjustment in the early phases of rehabilitation, above injury characteristics. Findings highlighted the vulnerable subgroups that are more likely to initially endorse negative appraisals, which may influence clinical practice and provides an avenue for further research.
\end{abstract}

\section{Introduction}

Spinal cord injury (SCI) is a traumatic event resulting in significant physical, psychological and social challenges. The consequences of an SCI results in the disruption of an individual's life and triggers the beginning of an individual's life-long psychological adjustment [1]. Variability in adjustment is evident within the SCI population [2] and cannot wholly be predicted by biomedical markers, such as physical impairment or injury severity [3]. This indicates

Kevin Jones

kevin.jones@buckshealthcare.nhs.uk

1 Department of Clinical Psychology, The National Spinal Injuries Centre, Stoke Mandeville Hospital, BHT, Aylesbury, UK the importance of psychological and social factors in the process of psychological adjustment.

Contemporary models of the process of psychological adjustment after chronic illness and disability are informed by Lazarus and Folkman's Transactional Model of Stress and Coping [4], which has been adapted and applied to adjustment following an SCI [5]. Duff and Kennedy [6] proposed that stress occurs when an individual identifies an imbalance between perceived situational demands (primary cognitive appraisals) and perceived coping resources (secondary cognitive appraisals). A stressful situation is constantly re-appraised by the individual based on increased information [6]. Thus, in terms of an individual's emotional and psychological reaction to a stressful event such as an SCI, their subjective perception of the situation is considered to be more important than the event itself [4]. Cognitive models also emphasise the complex, multifaceted, non-linear 
adjustment process and importance of biopsychosocial factors that mediate cognitive appraisals [7].

Kennedy et al. [8] examined the relationship between appraisals, coping and psychological health. Findings reported that appraisals and coping strategies measured at 12 weeks were significantly related to psychological distress 2 years post-injury. Further analysis by Kennedy et al. [9] reported that appraisals and coping strategies early in rehabilitation were significant predictors of depression at 21 years post-injury and a significant contributing factor of premature mortality. This indicates that cognitive appraisals made in the initial phase of rehabilitation influence longterm psychological and physical outcomes [10]. Kennedy et al. [11] found that individuals that endorsed negative appraisals reported lower levels of functional independence. Individuals who appraise their injury as a loss or threat may adopt a resigned behavioural response, thus increasing an individual's dependency on others for health needs [11]. Therefore, appraisals formed in acute rehabilitation settings impact psychological and functional outcomes [8, 11], and are possible indicators of adjustment and engagement during rehabilitation.

Dean and Kennedy [3] developed the Appraisals of Disability Scale Primary and Secondary Scale (ADAPSS) in order to encapsulate important stressors specific to the SCI population, over and above what is captured by more generic measures of cognitive appraisals such as the Appraisals of Life Events Scale (ALE) [12]. The ADAPSS long form measures six key constructs: 'fearful despondency', 'overwhelming disbelief', 'determined resolve', 'negative perceptions of disability', 'growth and resilience' and 'personal agency'. The authors identified two superordinate factors that encompassed all six key constructs: catastrophic negativity and determined resilience [3]. Preliminary analysis of the ADAPSS long form reported acceptable validity within a community-based sample and predicted $10 \%$ more variance in concurrent depression compared to non-SCI measures [3]. Results demonstrated that cognitive appraisals were positive predictors of distress, whereas demographic factors and injury characteristics were poor predictors of adjustment [3]. Research has demonstrated the importance of appraisals in the early phase of rehabilitation, therefore an increased understanding of appraisals may facilitate more effective identification and psychological treatment of maladaptive appraisals $[3,7,8,13]$.

At the National Spinal Injuries Centre (NSIC), Stoke Mandeville Hospital, all new admissions complete the Needs Assessment Checklist (NAC) [14]. The NAC forms part of the structured multidisciplinary framework of rehabilitation, which assesses and defines an individual's knowledge and skills across several domains of rehabilitation needs. Integrated into the psychological health domain was the ADAPSS short form (ADAPSS-SF), developed by
Dean and Kennedy, to be used in a clinical setting as an indicator of negative appraisals. Exploratory preliminary analysis of the ADAPSS-SF by Mignogna et al. [15] in a community-based sample of veterans with SCI supports the internal validity and demonstrated a coherent two-factor structure: resilience and loss.

The current paper aims to explore the factor structure and validity of the ADAPSS-SF in an acutely injured population. Additionally, the paper aims to explore the relationship between cognitive appraisals, demographic variables, injury characteristics and concurrent psychological distress.

\section{Methods}

The current research reports on retrospective cross-sectional data from inpatients at the NSIC. Individuals included within the sample were new admissions to the NSIC from February 2015 to January 2018. All patients were acutely injured; causes of injury were both traumatic and sustained through illness or disease.

\section{Measures}

All psychological data (i.e. ADAPSS-SF and HADS) used were routinely collected on the Psychological Health domain of the NAC [14]. Individual profiles, also collected from the NAC [14], included gender, ethnicity, civic status, age at injury, cause of injury and level of injury.

\section{ADAPSS-SF (developed from ADAPSS long form [3])}

This is an SCI-specific measure of cognitive appraisals following SCI. ADAPSS-SF is a six-item measure. Higher scores indicate more negative appraisals, which are indicative of negative adjustment and maladaptive coping strategies. Resilience items 'determined resolve', 'growth and resilience' and 'personal agency' are reverse coded; therefore by endorsing positive appraisals, individuals obtain a lower score. Internal consistency, validity and a two-factor structure (loss and resilience) of the ADAPSS-SF has been found within a community-based SCI population [15]. Clinical cutoff scores will be determined by statistical analysis from the current sample. Cronbach's $\alpha$ coefficient was 0.72 for the current study.

\section{Hospital Anxiety and Depression Scale (HADS) [16])}

HADS is a 14-item scale that measures the state of anxiety and depression. Higher scores are indicative of increased anxiety and depression, with a score of 11 or more indicating clinically significant distress, and a score of $8-10$ indicating possible clinical distress. The HADS has been 
validated within the SCI population [5]. Cronbach's $\alpha$ coefficient was 0.84 for HADS anxiety subscale and 0.76 for HADS depression for the current study.

\section{Statistical analysis}

IBM SPSS version 17.0 was used to conduct all analyses. Descriptive statistics were used to report sample demographics, injury characteristics, appraisals and mood. To determine the factor structure of the ADAPSS-SF, Principle-Components Analysis (PCA) with oblique rotation (oblimin with Kaiser Normalisation) was conducted. Correlational analysis and forced entry hierarchical stepwise regression analysis were conducted to determine the relationship between appraisals and mood. The data satisfied the assumptions of multicollinearity, outliers, linearity, normality and homoscedasticity.

\section{Results}

\section{Participant characteristics}

The sample included 371 participants that were predominantly White-British males and sustained SCI through injury, illness or disease (see Table 1). The mean age of the participants at the time of SCI was 53 years, ranging from 15 to 91 years.

\section{Descriptive statistics for appraisals and mood}

ADAPSS-SF total scores range from 6 to 36. Each item is scored on a 6-point likert scale, thus the sample utilised the full range of individual item scores. Standardisation and norming procedures were conducted to establish a clinical cutoff score constructed on corresponding percentile ranks, with the 85th percentile categorised as significant [17]. For the total ADAPSS-SF, individuals that scored 27 or more are categorised as significant; therefore a score of 27 or more is proposed as a clinical indication of maladaptive appraisals within acute SCI populations (see Table 2). Overall, the sample endorsed a number of resilient and adaptive appraisals in conjunction with negative, maladaptive appraisals. HADS scores indicate significant levels of anxiety and depression in the acute phase of rehabilitation following an SCI (see Table 2).

\section{Factor analysis}

PCA with oblique rotation (oblimin with Kaiser Normalisation) was conducted on ADAPSS-SF six-items. The current study has a ratio of 63 individuals to each item, which surpasses the recommendations within the literature
Table 1 Participant demographics and injury characteristics

\begin{tabular}{|c|c|}
\hline Characteristics & Descriptor, $n(\%)$ \\
\hline \multicolumn{2}{|l|}{ Sex } \\
\hline Male & $261(70)$ \\
\hline Female & $110(30)$ \\
\hline \multicolumn{2}{|l|}{ Civic status } \\
\hline Married & $142(38.3)$ \\
\hline Single & $83(22.4)$ \\
\hline Living with partner/engaged/relationship & $20(5.4)$ \\
\hline Divorced/separated & $19(5.1)$ \\
\hline Widowed & $17(4.6)$ \\
\hline Not stated & $90(24.3)$ \\
\hline \multicolumn{2}{|l|}{ Ethnicity } \\
\hline White-British & $260(70.1)$ \\
\hline White-other & $20(5.3)$ \\
\hline Black or Black British & $18(4.9)$ \\
\hline Asian or Asian British & $13(3.4)$ \\
\hline Mixed & $8(2.2)$ \\
\hline Other & $1(0.3)$ \\
\hline Not stated & $51(13.7)$ \\
\hline \multicolumn{2}{|l|}{ Cause of injury } \\
\hline Non-traumatic & $165(44.5)$ \\
\hline Fall & $112(30.2)$ \\
\hline RTA & $62(16.7)$ \\
\hline Sport & $22(5.9)$ \\
\hline Assault & $6(1.6)$ \\
\hline Other & $4(1.1)$ \\
\hline \multicolumn{2}{|l|}{ AISA grade } \\
\hline A & $79(21.3)$ \\
\hline B & $56(15.1)$ \\
\hline $\mathrm{C}$ & $106(28.6)$ \\
\hline $\mathrm{D}$ & $130(35.0)$ \\
\hline \multicolumn{2}{|l|}{ Level of injury } \\
\hline Cervical & $179(48.2)$ \\
\hline Thoracic & $143(38.5)$ \\
\hline Lumbar & $45(12.1)$ \\
\hline Sacrum & $4(1.1)$ \\
\hline \multicolumn{2}{|l|}{ Injury type } \\
\hline Complete tetraplegia & $28(7.5)$ \\
\hline Complete paraplegia & $51(13.7)$ \\
\hline Incomplete tetraplegia & $151(40.7)$ \\
\hline Incomplete paraplegia & $141(38.0)$ \\
\hline
\end{tabular}

summarised in Kristine et al. [18]. Kaiser-Meyer-Olkin measure of sampling adequacy was 0.78, above the recommended 0.6, and Bartlett's test of sphericity was significant $\left.\chi^{2}(15)=419.24, p=0.00\right)$, indicating all items suitable for PCA. Two factors, both with three items, were identified; resilience and loss. Both factors had eigenvalues above 1 [19] and accounted for $60.7 \%$ of the variance 
Table 2 Descriptive statistics for appraisals and mood scales

\begin{tabular}{llllll}
\hline Measures & $M(\mathrm{SD})$ & Score indicating clinical case & Percentage of sample & Score indicating possible case & Percentage of sample \\
\hline ADAPSS-SF & $19.21(6.96)$ & 27 or more & $14.80 \%$ & - & - \\
HADS anxiety & $6.10(4.70)$ & $>11$ & $18.40 \%$ & $8-10$ & $12.30 \%$ \\
HADS depression & $6.30(4.28)$ & $>11$ & $18.30 \%$ & $8-10$ & $19.20 \%$ \\
\hline
\end{tabular}

Table 3 Standardised pattern of coefficients for un-rotated and rotated factors of the ADAPSS$\mathrm{SF}$

\begin{tabular}{lllllll}
\hline Components & Un-rotated & & & Rotated & Communalities \\
\cline { 2 - 3 } & Factor 1 resilience & Factor 2 loss & & Factor 1 resilience & Factor 2 loss & \\
\hline Q5 & 0.801 & 0.254 & $\mathbf{0 . 8 2 1}$ & -0.054 & 0.631 \\
Q3 & 0.792 & 0.348 & $\mathbf{0 . 7 7 0}$ & 0.060 & 0.644 \\
Q6 & 0.751 & 0.274 & $\mathbf{0 . 7 5 5}$ & -0.008 & 0.565 \\
Q2 & 0.203 & 0.791 & -0.109 & $\mathbf{0 . 8 3 2}$ & 0.659 \\
Q1 & 0.325 & 0.811 & 0.025 & $\mathbf{0 . 8 0 2}$ & 0.639 \\
Q4 & 0.522 & 0.643 & 0.327 & $\mathbf{0 . 5 2 0}$ & 0.505 \\
\hline
\end{tabular}

Bolded values represent loadings of 0.5 or more.

Table 4 Pearson's correlations between ADAPSS-SF total, ADAPSS-SF factors (resilience and loss), HADS anxiety and HADS depression

\begin{tabular}{lllll}
\hline & ADAPSS-SF factor 1 (resilience) & ADAPSS-SF factor 2 (loss) & HADS-anxiety & HADS-depression \\
ADAPSS-SF total & $0.811^{* *}$ & $0.864^{* *}$ & $0.597^{* *}$ & $0.633^{* *} \mathrm{~d}$ \\
ADAPSS-SF factor 1 (resilience) & - & $0.438^{* *}$ & $0.398^{* *}$ & $0.520^{* *}$ \\
ADAPSS-SF factor 2 (loss) & - & - & $0.605^{* *}$ & $0.597^{* *}$ \\
HADS-anxiety & - & - & - & $0.649^{* *}$ \\
\hline
\end{tabular}

*** $p=0.01$ (one tailed)

cumulatively. Factor one represented appraisals of resilience and accounted for $43.3 \%$ of the variance with an eigenvalue of 2.60. Factor two represents appraisals of loss and accounted for $17.3 \%$ of the variance with an eigenvalue of 1.04. Each item loaded heavily onto one factor (as shown in Table 3) and the two factors moderately correlated ( $r=$ 0.38). Cronbach's $\alpha$ coefficients were 0.70 and 0.63 for factors 1 and 2, respectively, indicating good reliability.

\section{Relationship between the ADAPSS-SF factors, HADS subscales and sample characteristics}

ADAPSS-SF total and both factors (resilience and loss) were significantly positively correlated to both the HADS subscales (see Table 4). Moderate to strong correlations indicate that the individuals who negatively appraise their SCI, by endorsing loss items and rejecting resilience items, reported higher levels of concurrent anxiety and depression. ADAPSS-SF factors (resilience and loss) were unrelated to civic status, ethnicity and injury level. Weak correlations demonstrated that females and individuals older at the time of injury were more likely to endorse loss items and less likely to endorse resilience items. Individuals with AIS A injuries were less likely to endorse resilience factors and those with an SCI acquired through assault were more likely to endorse loss items. Findings highlight potentially vulnerable groups (i.e. females, individuals older at the time of injury, individuals with AIS A injuries, individuals whose SCI was acquired through assault) that may be more likely to negatively appraise their injury in acute rehabilitation.

\section{Hierarchical regression model}

Hierarchical stepwise regression analysis was conducted to explore whether ADAPSS-SF factors were predictors of variance in concurrent anxiety and depression. Resilience and loss factors were significant predictors of concurrent depression and accounted for $44.2 \%$ of the variance. Gender, resilience and loss factors were all significant predictors of concurrent anxiety and accounted for $36.9 \%$ of the variance. Standardised $\beta$ coefficients demonstrate that loss factor has the biggest impact for concurrent anxiety. Regression analysis demonstrates that cognitive appraisals are more significant predictors of concurrent depression and anxiety than biological markers (Table 5).

\section{Discussion}

Study findings support a coherent two-factor structure and validity of the ADAPSS-SF in an acutely injured SCI 
Table 5 Significant predictors of concurrent depression and concurrent anxiety from hierarchical regression analysis

\begin{tabular}{|c|c|c|c|c|c|c|}
\hline \multirow[t]{2}{*}{ Variable } & \multirow[t]{2}{*}{ Adjusted $R$ squared } & \multicolumn{2}{|c|}{$\begin{array}{l}\text { Unstandardised } \\
\text { coefficients }\end{array}$} & \multicolumn{3}{|c|}{$\begin{array}{l}\text { Standardised } \\
\text { coefficients }\end{array}$} \\
\hline & & $B$ & SE & $B$ & $t$ & $P$ \\
\hline Final model for concurrent depression & 0.442 & & & & & \\
\hline (Constant) & & -2.140 & 0.632 & & -3.38 & $0.001^{* *}$ \\
\hline Gender & & 0.649 & 0.368 & 0.070 & 1.77 & 0.078 \\
\hline ADAPSS-SF factor 1 (resilience) & & 0.391 & 0.047 & 0.361 & 8.33 & $0.000^{* *}$ \\
\hline ADAPSS-SF factor 2 (loss) & & 0.406 & 0.043 & 0.408 & 9.35 & $0.000^{* *}$ \\
\hline Final model for concurrent anxiety & 0.369 & & & & & \\
\hline (Constant) & & -2.858 & 0.723 & & -3.95 & $0.000^{* *}$ \\
\hline Gender & & 1.159 & 0.420 & 0.116 & 2.76 & $0.006^{* *}$ \\
\hline ADAPSS-SF factor 1 (resilience) & & 0.241 & 0.054 & 0.207 & 4.50 & $0.000^{* *}$ \\
\hline ADAPSS-SF factor 2 (loss) & & 0.491 & 0.050 & 0.459 & 9.90 & $0.000^{* * *}$ \\
\hline
\end{tabular}

population. The factor structure found in the current study reflects Dean and Kennedy's [3] findings from the ADAPSS long-form validation study which also reported two superordinate factors: catastrophic negativity and determined resilience. Findings are also consistent with previous exploratory factor analysis of ADAPSS-SF in a community-based sample [15]. In contrast to the findings by Mignogna et al. [15], ADAPSS-SF's resilience factor explained a larger percentage of variance for the current sample of acutely injured individuals, whereas the loss factor explained more of the variance in the communitybased sample. In the current study, the single loss item 'negative perception of disability' demonstrated weaker correlation coefficients compared to all other items and was considerably lower compared to community-based samples [15]. Furthermore, longitudinal analysis of 21 years post-injury reported significantly lower scores for this key construct [9]. Taken together, an interpretation of these findings is that in the early phases of adjustment to an SCI, there is a high degree of variability in terms of who endorses 'negative perceptions of disability'; and while significant, this factor appears to be a relatively weak indicator compared to other loss appraisals. Over time, however, this factor increasingly becomes a stronger indicator of maladjustment, highlighting the importance of reinforcing a positive and balanced perception of disability in the initial phase of rehabilitation. Peer support or mentoring schemes have been found to positively influence an individual's negative perceptions of disability and self-management [20]. Further exploratory analysis is needed to replicate such findings in acutely injured samples and to better understand the variability in cognitive appraisal across different cohorts.

Cognitive appraisals made in the initial phase of rehabilitation accounted for a significant variance in concurrent anxiety and depression, over and above injury characteristics or biological markers. These findings are consistent with prior research in community-based samples [3] and provides support for the use of cognitive models of adjustment following an SCI. Prevalence of anxiety and depression was higher in the current sample compared to the general UK population [21], which is a comparable finding to previous research in acute rehabilitation settings [22], and slightly higher than community samples $[8,9,13$, $23,24]$. With the knowledge that mood and adjustment during the acute phase of recovery is a predictor of longterm adjustment and psychological distress [9], the findings of the current study highlight the importance of early psychological intervention to support psychological adjustment and minimise the risk of long-term psychological distress $[25,26]$. Establishing a clinical cutoff for the ADAPSS-SF will better enable clinicians to identify those at risk of developing more entrenched, negative perceptions of their situation.

Findings highlighted four vulnerable groups that were more likely to negatively appraise their injury in the acute phase: females, individuals older at the time of injury, individuals with AIS A injuries and individuals whose SCI was acquired through an assault. In comparison with Mignogna et al. [15], complete injuries were less likely to endorse resilience items, thus indicating significance in the acute phase of rehabilitation. Community-based research demonstrates that females are more likely to negatively appraise their injury, and utilise maladaptive, emotion-focused coping strategies [3, 27]. Furthermore, individuals who sustain their injury later in life are significantly more likely to report higher levels of depression or anxiety, lower life satisfaction, lower purpose in life, less usage of adaptive coping strategies, and more likely to appraise their injury as a loss [3, 27]. Older adults have increased frailty, dependency on others for assistance and increased risk of secondary complications, 
which may account for the increased negative appraisals $[25,27]$. Prevalence of sustaining an SCI later in life and the percentage of females sustaining SCI's have increased in recent years [28], highlighting the need for further research and targeted therapeutic interventions for these vulnerable subgroups.

\section{Limitations}

Due to the cross-sectional design of the study, a causal relationship between appraisals and emotional adjustment cannot be determined. Adjustment is a transactional process, rather than an isolated experience, thus future longitudinal data should be collected to investigate the causal relationship between cognitive appraisals made in the initial phase of rehabilitation on rehabilitation outcomes and longterm adjustment.

Overall, the study provides evidence to support the importance of cognitive appraisals on mood during the initial stages of rehabilitation and use of the ADAPSS-SF as a clinical measure of appraisals in acute rehabilitation. Current findings have also highlighted some vulnerable groups following SCI, such as females and older adults, which require a greater emphasis in the research literature. Finally, it should be noted that a considerable percentage of individuals within the sample employed adaptive, positive appraisals highlighting a focus on adaptive and resilient coping resources during early rehabilitation. The current study provides many avenues for further research, including further exploration of the specific experiences of vulnerable groups in the early phases of adjustment, and measuring changes in cognitive appraisal over time and use of the ADAPSS-SF in a variety of clinical cohorts.

Acknowledgements We would like to thank all the patients and staff at The National Spinal Injuries Centre, Stoke Mandeville Hospital, who participated in this research, and notably the Department of Clinical Psychology, who manages and coordinates the NAC and goal planning programme at the National Spinal Injuries Centre.

Author contributions RE was responsible for designing the study, conducting data analysis, interpreting the results, writing and revising the manuscript, updating reference lists and creating summary tables, and approval of the final manuscript. KJ was responsible for designing the study, overseeing data collection, interpreting the results, writing and revising the manuscript, and approval of the final manuscript. JD was responsible for developing the research conception, overseeing data collection contributed to the revision of the manuscript and approval of the final manuscript. All authors agreed to be accountable for all aspects of the work in ensuring that questions related to the accuracy or integrity of any part of the work are appropriately investigated and resolved.

\section{Compliance with ethical standards}

Conflict of interest The authors declare that they have no conflict of interest.

\section{References}

1. Westie KS. Psychological aspects of spinal cord injury. Clin Prosthet Orthot. 1987;11:4.

2. Chevalier Z, Kennedy P, Sherlock O. Spinal cord injury, coping and psychological adjustment: a literature review. Spinal Cord. 2009;47:778-82.

3. Dean RE, Kennedy P. Measuring appraisals following acquired spinal cord injury: a preliminary psychometric analysis of the appraisals of disability. Rehabil Psychol. 2009;54: 222-31.

4. Lazarus RS, Folkman S. Coping and adaptation. In: W. D. Gentry (Ed.), The handbook of behavioural medicine. New York: Guilford. 1984. p. 282-325.

5. Galvin LR, Godfrey HP. The impact of coping on emotional adjustment to spinal cord injury (SCI): review of the literature and application of a stress appraisal and coping formulation. Spinal Cord. 2001;39:615.

6. Duff J, Kennedy P. Spinal cord injury. In: Llewelyn SP, Kennedy P, editors. Handbook of clinical health psychology. Chichester: John Wiley and Sons; 2003. p. 251-78.

7. Holroyd KA, Lazarus RS. Stress, coping and somatic adaptation. In: L Goldberger and S Breznitz (Eds), Handbook of stress: theoretical and clinical aspects. New York: 1982. p. 21-35.

8. Kennedy P, Lude P, Elfström ML, Smithson E. Cognitive appraisals, coping and quality of life outcomes: a multi-centre study of spinal cord injury rehabilitation. Spinal Cord. 2010;48:762-9.

9. Kennedy P, Kilvert A, Hasson L. A 21-year longitudinal analysis of impact, coping, and appraisals following spinal cord injury. Rehabil Psychol. 2016;61:92.

10. Kennedy P, Evans M, Sandhu N. Psychological adjustment to spinal cord injury: the contribution of coping, hope and cognitive appraisals. Psychol Health Med. 2009;14:17-33.

11. Kennedy P, Smithson E, McClelland M, Short D, Royle J, Wilson C. Life satisfaction, appraisals and functional outcomes in spinal cord-injured people living in the community. Spinal Cord. 2010;48:144.

12. Ferguson E, Matthews G, Cox T. The appraisal of life events (ALE) scale: reliability and validity. $\mathrm{Br} \mathrm{J}$ Health Psychol. 1999;4:97-116.

13. Kennedy P, Rogers BA. Anxiety and depression after spinal cord injury: a longitudinal analysis. Arch Phys Med Rehabil. 2000;81:932-7.

14. Kennedy P, Hamilton LR. The needs assessment checklist: a clinical approach to measuring outcome. Spinal Cord. 1999;37:136-9.

15. Mignogna J, Christie AJ, Holmes SA, Ames H. Measuring disability-associated appraisals for veterans with spinal cord injury. Rehabil Psychol. 2015;60:99.

16. Zigmond AS, Snaith RP. The hospital anxiety and depression scale. Acta Psychiatr Scand. 1983;67:361-70.

17. Sparrow SS, Balla DA, Cicchetti DV, Harrison PL, Doll EA. Vineland adaptive behavior scales. Circle Pines, MN: American Guidance Service.

18. Hogarty KY, Hines CV, Kromrey JD, Ferron JM, Mumford KR. The quality of factor solutions in exploratory factor analysis: the influence of sample size, communality, and overdetermination. Educ Psychol Meas. 2005;65:202-26.

19. Kaiser HF. The application of electronic computers to factor analysis. Educ Psychol Meas. 1960;20:141-51.

20. Munce SE, Webster F, Fehlings MG, Straus SE, Jang E, Jaglal SB. Perceived facilitators and barriers to self-management in individuals with traumatic spinal cord injury: a qualitative descriptive study. BMC Neurol. 2014;14:48.

21. Mental Health Foundation. Fundamental facts about mental health 2016. London, UK: Mental Health Foundation; 2016. 
22. Kennedy P, Nolan M, Smithson E. Psychological adjustment to spinal cord injury in Ireland: quality of life, appraisals and coping. Ir J Psychol. 2011;32:116-29.

23. Schönenberg M, Reimitz M, Jusyte A, Maier D, Badke A, Hautzinger M. Depression, posttraumatic stress, and risk factors following spinal cord injury. Int J Behav Med. 2014;21:169-76.

24. Williams RT, Wilson CS, Heinemann AW, Lazowski LE, Fann JR, Bombardier CH. PRISMS Investigators. Identifying depression severity risk factors in persons with traumatic spinal cord injury. Rehabil Psychol. 2014;59:50-6.

25. Bombardier CH, Ehde DM, Stoelb B, Molton IR. The relationship of age-related factors to psychological functioning among people with disabilities. Phys Med Rehabil Clin N Am. 2010;21:281-97.

26. Kraft R, Dorstyn D. Psychosocial correlates of depression following spinal injury: a systematic review. J Spinal Cord Med. 2015;38:571-83.

27. Geyh S, Kunz S, Müller R, Peter C. Describing functioning and health after spinal cord injury in the light of psychologicalpersonal factors. J Rehabil Med. 2016;48:219-34.

28. White NH, Black NH. Spinal cord injury (SCI) facts and figures at a glance. National Spinal Cord Injury Statistical Center, Facts and Figures at a Glance. Birmingham, AL: University of Alabama at Birmingham, 2016. 